\title{
Parallel Tensile Testing of Single-crystal Silicon Microstructures with Integrated Piezoresistive Strain Gauges
}

\author{
Akio Uesugi, Yoshikazu Hirai, Toshiyuki Tsuchiya, ${ }^{*}$ and Osamu Tabata \\ Department of Micro Engineering, Kyoto University, \\ Kyotodaigaku-Katsura C3, Nishikyo-ku, Kyoto 615-8540, Japan
}

(Received March 29, 2018; accepted July 23, 2018)

Keywords: single-crystal silicon, MEMS, tensile testing, parallel testing, strain gauge

A parallel testing method has been proposed with integrated strain gauges based on the piezoresistive effect to shorten the testing time for investigating tensile-mode fatigue characteristics of single-crystal silicon (SCS). The method allows a high loading frequency ( $\sim 110$ $\mathrm{Hz}$ ) owing to the high stiffness of the testing system and reduces the testing time per specimen by parallelization. Five specimens were integrated on a testing chip and subjected to a pulling force simultaneously using a piezoelectric actuator. The parallel portion of the specimen was $2.5 \mu \mathrm{m}$ wide, $22 \mu \mathrm{m}$ thick, and $120 \mu \mathrm{m}$ long. The strain gauge connected to each gauge part consisted of an array of five straight beams $100 \mu \mathrm{m}$ in length and $2.5 \mu \mathrm{m}$ in width. The oneactive-gauge bridge was used and three reference registers of identical shape were also placed in series on the chip. The strain gauges successfully monitored the force on each specimen with sufficiently low noise for testing. The five specimens on the chip fractured at the same time, showing a tensile strength of $1.5 \mathrm{GPa}$. Because of the finite stiffness of the test system, there is coupling between the integrated specimens. However, the total displacement during parallel testing was sufficiently small $(\sim 15 \mu \mathrm{m})$ such that the system has the potential to operate with a loading frequency of $110 \mathrm{~Hz}$ for fatigue testing.

\section{Introduction}

Silicon is one of the standard materials used in micro-electromechanical systems (MEMS), and because MEMS devices require mechanical deformations of the structural materials to operate, many studies on silicon microstructure material properties have been reported. ${ }^{(1)}$ In addition, because silicon is a brittle material whose fracture strength is affected by many factors such as structure size and surface morphology, the fracture properties of these microstructures have also been investigated to design highly reliable MEMS devices. ${ }^{(2-9)}$ Recently, to improve the MEMS reliability for long-term operation, the fatigue properties of microstructures have become more important. The reliability is important especially for MEMS with resonators, such as vibratory gyroscopes and micromirrors, which are oscillated at frequencies higher than $1 \mathrm{kHz}$ and subjected to a large number of loading cycles in a short time.

*Corresponding author: e-mail: tutti@me.kyoto-u.ac.jp https://doi.org/10.18494/SAM.2018.1946 
There are many reports on the fatigue properties of silicon, ${ }^{(10-18)}$ which have indicated that the plots of stress vs number of cycles $(S-N)$ are generally fitted well to Paris' law. However, the measured fatigue life of silicon varied among the reports because of the different ambient conditions, testing methods, and testing structures used, and there is no consensus among researchers on the mechanism of fatigue crack growth in silicon. The "reaction layer fatigue"(10) and "mechanically induced subcritical cracking"(12) models have been proposed as crack growth models. These models indicate the importance of maximum stress as well as a range of stress intensity factors for crack growth. Moreover, compressive stress has been reported as a factor enhancing crack growth. Because tensile-mode fatigue testing allows the employment of a stress ratio in the range 0 to 1 at a fixed maximum stress, it is considered to be an effective method of revealing the crack growth mechanism.

Unlike bending-fatigue testing, there are few reports on tensile-mode fatigue testing. One of the difficulties in employing tensile-mode fatigue testing is its low loading frequency. The loading frequency determines the testing time, which in turn limits the number of loading cycles achievable in the fatigue testing. Because fatigue testing for silicon microstructures requires more than $10^{7}$ cycles and a sufficient number of samples for a statistical analysis that considers deviations in fatigue life because of its brittleness, a low loading frequency is unfavorable. Table 1 shows the reported methods for tensile-mode fatigue testing, ${ }^{(13-18)}$ wherein the loading frequencies were typically lower than $100 \mathrm{~Hz}$. Contrasted with ultrahigh-cycle bending-fatigue testing that is often performed with resonant vibrations at frequencies higher than $10 \mathrm{kHz},{ }^{(10,11)}$ the loading frequencies of tensile-mode fatigue testing are so low that the testing is often limited to $10^{6}$ cycles.

The maximum loading frequency in tensile-mode fatigue testing depends strongly on the performance of loading actuators. As shown in Table 1, external actuators (e.g., piezoelectric actuators) are often used to apply tensile force. Because silicon microstructures exhibit high tensile strengths, it is difficult to generate a force large enough to break the specimen using an integrated MEMS actuator. The maximum loading frequency is also affected by the stiffness of the testing system, which comprises the specimen, actuator, load sensor, and other components of the test setup. The amplitudes of external actuators generally decrease as the actuation frequencies increase; for example, the amplitudes of the piezoelectric actuators decrease depending on the time constants of the actuators and the output impedances of the driving circuits. Therefore, it is difficult to increase the loading frequency in a microscale testing

Table 1

Reported tensile-mode fatigue testing methods for silicon microstructures.

\begin{tabular}{lcccccc}
\hline Authors (Year) & Ref. No. & Material & Loading & Sensing & Freq. (Hz) & Cycles \\
\hline Namazu (2009) & 13 & sc-Si & Piezo & Load cell & $10-200$ & $10^{9}$ \\
Tsuchiya (2010) & 14 & sc-Si & Piezo & Load cell & 40 & $10^{6}$ \\
Ando (2001) & 15 & sc-Si & DC servo & Load cell & 10 & $10^{6}$ \\
Bagdahn (2003) & 16 & poly-Si & Loud speaker & Load cell & $50-6000$ & $10^{9}$ \\
Kamiya (2008) & 17 & poly-Si & Piezo & Load cell & 100 & $10^{8}$ \\
Kapels (2000) & 18 & poly-Si & Thermal & Optical & - & $10^{6}$ \\
\hline
\end{tabular}


system that requires a large displacement for testing. The microscale testing system usually has a low stiffness because of a force sensor of high sensitivity.

To shorten the time for investigating the tensile-fatigue properties of single-crystal silicon (SCS) microstructures, we have proposed a parallel testing method with integrated piezoresistive strain gauges. ${ }^{(19)}$ The method increases the loading frequency because of its high stiffness and shortens the testing time per specimen by parallelization. The testing devices consisting of a specimen and an integrated strain gauge were arrayed on a chip and simultaneously subjected to a tensile force using a piezoelectric actuator. Because the strain gauge possesses a linear output with low noise and is operated with a simple readout circuit, the parallelization of the testing is easy. In this paper, we report the validation of the proposed integrated strain gauges and testing system through tensile testing.

\section{Parallel Tensile Testing}

\subsection{Chip and device design}

Figure 1 shows schematics of a chip for the proposed method. The chip was designed to be fabricated from silicon-on-insulator (SOI) wafers. As shown in Fig. 1(a), the chip with dimensions of $7 \times 15 \mathrm{~mm}^{2}$ was composed of an array of five testing devices. In each device, one end of a tensile specimen was connected to an integrated force sensor fixed to the chip frame and the other end was connected to a common movable plate, called a loading shuttle, which was supported with four beams of $75 \mu \mathrm{m}$ width and $1 \mathrm{~mm}$ length consisting of both the

$\mathrm{Si} \square$ Metal $\square \mathrm{SiO}_{2}$

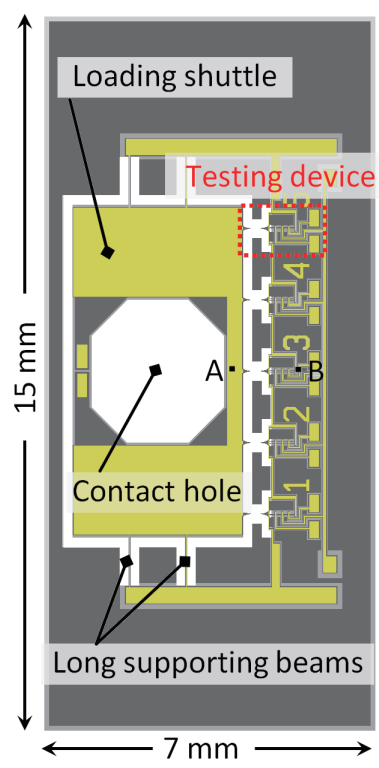

(a)

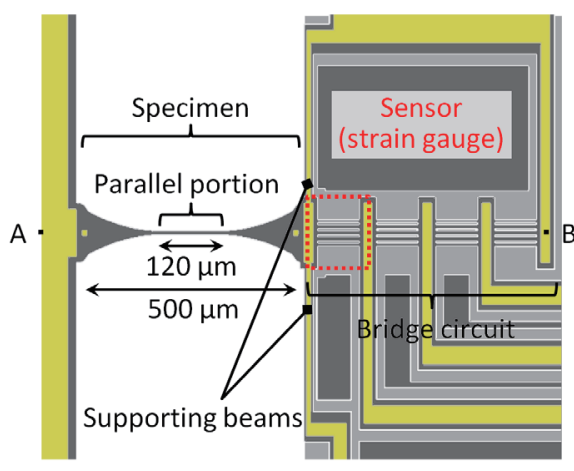

(b)

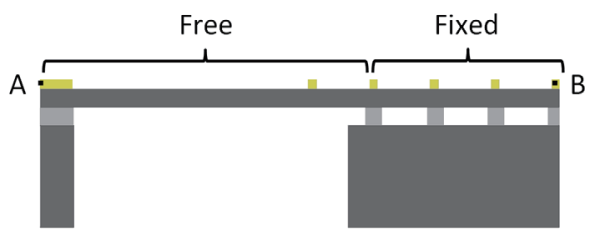

(c)

Fig. 1. (Color online) Chip design for parallel tensile testing. (a) Top view of whole chip. (b) Top view of single device. (c) A-B cross section. 
device and handle layers. The loading shuttle has a through hole at the center, called a contact hole. The tensile force was applied by pushing the shuttle using an external piezoelectric actuator.

The specimens were aligned along the $<110>$ orientation on SOI wafers with a surface orientation of (100), which is the standard orientation for MEMS. The specimens consisted of a parallel portion subjected to tensile testing, with the shoulders on the ends rounded to prevent stress concentration. The parallel portion was designed to be $2.5 \mu \mathrm{m}$ wide, $22 \mu \mathrm{m}$ thick, and $120 \mu \mathrm{m}$ long. Each end of the specimen had a metal pattern of $10 \mu \mathrm{m}$ square. Metal patterns with an intrapattern distance of $500 \mu \mathrm{m}$ were used as reference points for elongation measurement using digital image correlation (DIC).

The dimensions of the specimens were chosen to verify the proposed system. The thickness was decided considering its fabrication yield, since the thinner specimen may have a low yield. The length of the specimen is the same as that in our previous work for quasi-static tensile testing for strength comparison strength. In the literature, shorter and thinner specimens are often used for fatigue testing since a smaller elongation and a lower stiffness are suitable for the high testing frequency, as well as parallel testing.

\subsection{Strain gauge}

A piezoresistive strain gauge made of SCS was used for the force sensor. Because SCS shows a piezoresistive effect, the properties of which depend on dopant concentration and crystallographic orientation, ${ }^{(20)}$ a large gauge factor is possible. Strain gauges have features suitable for the parallel testing and fatigue testing of microstructures in the following ways:

- Strain measurement: The direct measurement of strain is preferable in these tests, because the displacement or elongation is small but strain is large in tensile testing. The measurement of a large strain allows a high signal/noise ratio, linearity, and a simple readout circuit.

- Electrical measurement: Remote measurement is possible. It is easy to put the testing system into an oven or a vacuum chamber.

- Small footprint: The sensor can be designed with a small footprint and is easy to connect to a specimen.

The strain gauge in the device consists of a parallel array of five straight beams $100 \mu \mathrm{m}$ in length and $2.5 \mu \mathrm{m}$ in width. The width is identical to that of the specimen's parallel portion, as shown in Fig. 1(b). The strain gauge was subjected to uniaxial tensile stress. Because the strain gauges had a much higher stiffness than the parallel portion, the gauge output was maintained within the linear output range, ${ }^{(21)}$ and the fracture at the strain gauge was avoided.

A bridge circuit for the strain gauge was also integrated to each specimen as a one-activegauge configuration, as shown in Fig. 1(b). The integration of reference gauges with identical designs to the strain gauge minimized the difference in gauge resistance and was suitable for temperature compensation. Because only the specimen was released from the substrate, as shown in Fig. 1(c), the same tensile force was applied on the strain gauge, which produced the linear bridge output. 


\subsection{Fabrication process}

The fabrication process consisted of patterning of an aluminum electrode, deep reactive ion etching (RIE) of the device and handle silicon layers, and etching of the buried oxide layer, as shown in Fig. 2(a). A fabricated chip is shown in Fig. 2(b), wherein the loading shuttle was fixed by short anchor beams patterned on the device layer to avoid the fracture of the specimens during fabrication. The anchor beams were cut using a laser microprocessing tool just prior to testing.

The thicknesses of the device, buried oxide, and handle layers of the SOI wafers were 22, 3 , and $400 \mu \mathrm{m}$, respectively. Because the tensile testing was conducted on (100) silicon along the $<110>$ direction, wafers with a device layer of p-type (boron-doped) silicon were used to give a high piezoresistive coefficient along the tensile axis. ${ }^{(20)}$ The resistivity was $0.01-0.02 \Omega \mathrm{cm}$. Because the fabrication process did not include a doping process for fabrication simplicity, the strain gauge has a low resistivity and therefore exhibits a lower gauge factor. However, the piezoresistive coefficient of heavily doped silicon possessed a smaller temperature coefficient, which resulted in a higher stability in testing.

\section{Testing System}

Figure 3 shows the setup for parallel tensile testing using the chip and Table 2 shows its specifications. Tensile force was applied via a 2-mm-diameter shaft called a loading pin fixed on the piezoelectric stage. The stage's static travel range is $80 \mu \mathrm{m}$ and the measured frequency response is shown in Fig. 4. It showed a sufficiently precise position control for tensile testing, but its travel range is too small to align the chip to a loading system. Therefore, for the $x$ - and $z$-axes alignment of the testing chip to insert the loading pin into the contact hole, the chip was

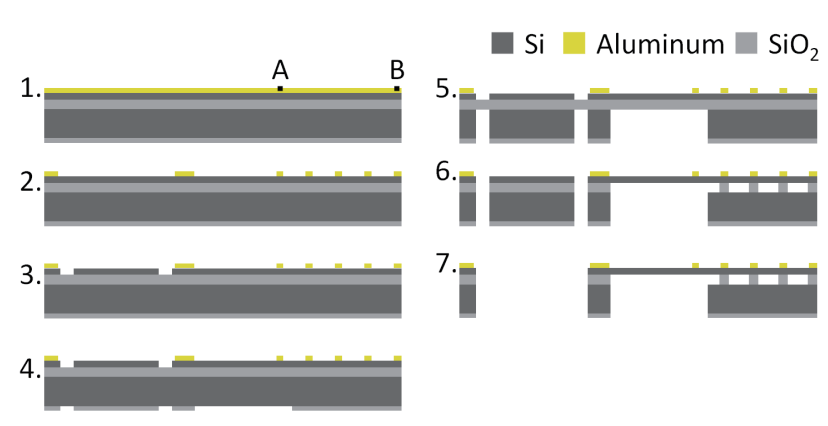

(a)

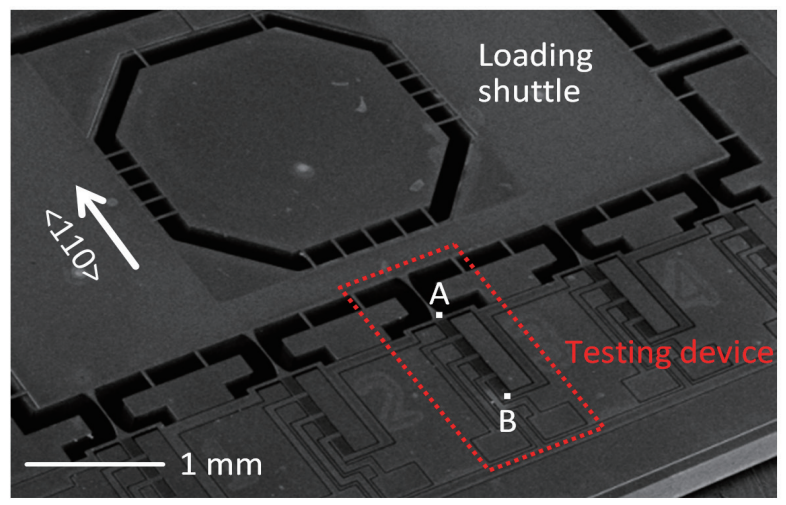

(b)

Fig. 2. (Color online) (a) Fabrication process: 1. aluminum deposition on top and $\mathrm{SiO}_{2}$ on back, 2. electrode patterning using wet etching, 3. Si deep RIE of device layer, 4. patterning of back-side $\mathrm{SiO}_{2}$ as a hard mask, 5. Si deep RIE of handle layer, 6. sacrificial etching, and 7. removal of dummy silicon structures and short anchor beams using a laser cutting tool. (b) Fabricated testing device after step 6. 


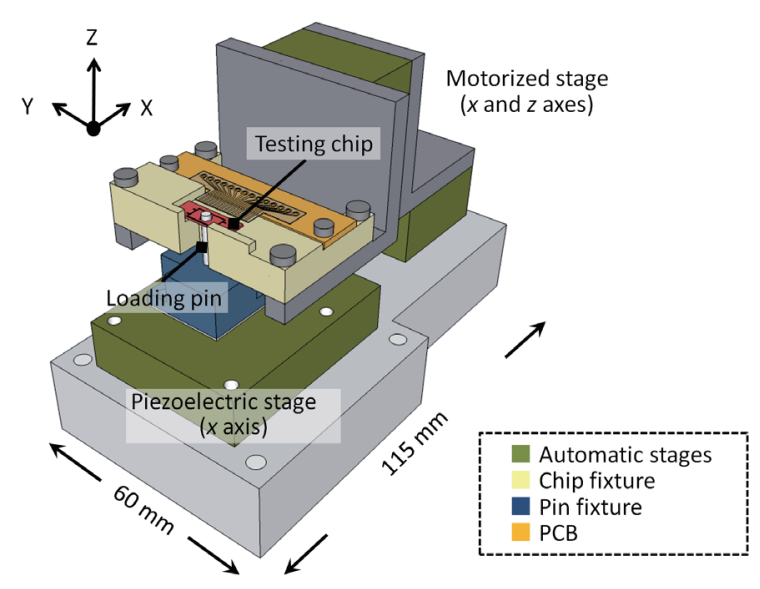

(a)

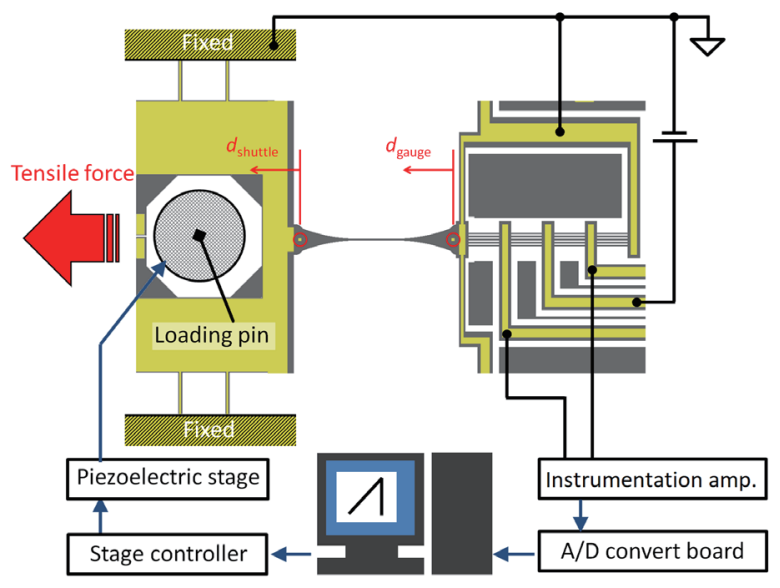

(b)

Fig. 3. (Color online) Setup for parallel tensile testing. (a) Schematics of setup. (b) Block diagram.

Table 2

Specifications of the testing system. The testing system components specified are the piezoelectric stage, the motorized stage, the loading pin, the microscope, and the CCD camera.

\begin{tabular}{ll}
\hline Piezoelectric stage & P-780.20 (PI) \\
\hline Travel range & $80 \mu \mathrm{Sm}$ \\
\hline Resolution & $10 \mathrm{~nm}$ \\
\hline Push/pull force capacity & $50 / 5 \mathrm{~N}$ \\
\hline Position detection & Embedded LVDT ${ }^{*}$ sensor \\
\hline Motorized stage & Picomotor 8351 (Newport) \\
\hline Travel range & $>5 \mathrm{~mm}$ \\
\hline Resolution & $30 \mathrm{~nm}$
\end{tabular}

*LVDT: linear variable differential transformer

\begin{tabular}{ll}
\hline Loading pin & SUS 304 shaft \\
\hline Diameter & $2 \mathrm{~mm}$ \\
\hline Microscope and CCD camera \\
\hline Microscope & ME600 (Nikon) \\
\hline Objective lens & $\times 10$ (NA: 0.30) \\
\hline Color CCD camera & XCL-U1000C (Sony) \\
\hline Image resolutions & H: 1600 pixels \\
& V: 1200 pixels \\
\hline Frame rate & $15 \mathrm{fps}$ \\
\hline Total pixel resolution & $0.73 \mu \mathrm{m} /$ pixel \\
\hline
\end{tabular}

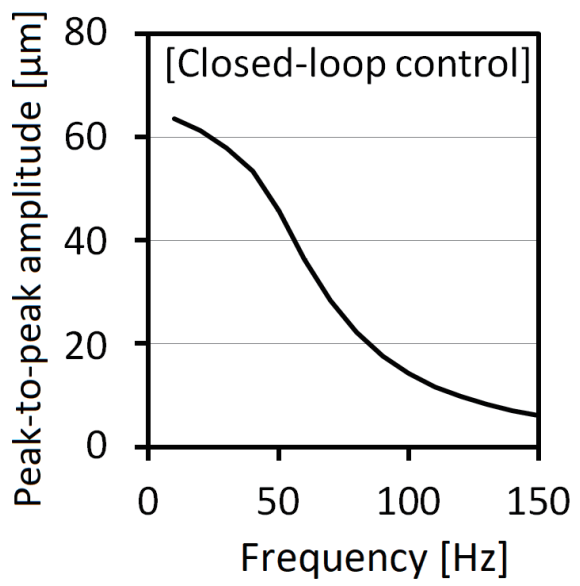

Fig. 4. Measured frequency response of the piezoelectric stage operated under dynamic closed-loop control. The peak-to-peak displacement amplitude against driving frequency was measured with the driving signal amplitude equivalent to a $64 \mu \mathrm{m}$ static displacement. 
mounted on two motorized $x$ - and $z$-stages, as shown in Fig. 3(a). The $y$-axis alignment was realized by the mechanical precision of the testing setup.

The testing setup shown in Fig. 3(a) was mounted on manual $x$ - and $y$-axis positioning stages and placed under an optical microscope. The optical microscope equipped with a chargecoupled device (CCD) camera was used for monitoring the chip alignment and tensile testing. Because the ambient humidity and temperature significantly affect the fatigue properties of silicon microstructures, the whole system was placed in a small clean booth, into which regulated air was supplied through a clean filter. The testing environment was kept constant in the range of $40-70 \mathrm{RH} \%$ (relative humidity $\%$ ) at $25^{\circ} \mathrm{C}$.

Figure 5 shows the equivalent circuit diagram of the chip. To simplify electrical wirings, the bridge voltage was supplied from one dc voltage source. Both ends of the specimens were connected to the ground, which was intended to prevent current flow on the specimens. The bridge circuits were electrically connected to the readout circuits as a block diagram shown in Fig. 3(b). The five bridge outputs were amplified using instrumentation amplifiers (INA 114, Texas Instruments) with a gain of 500. The output voltage was acquired using a personal computer.

DIC on the specimen images was used to measure the specimen elongation. The positions of two reference points on the specimen were detected to calculate the distance change between them, called a nominal elongation, in synchronizing with acquiring the bridge outputs. The purpose of the nominal elongation measurement was to calibrate the strain gauges, whose output may contain errors caused by fabrication imperfections and ambient humidity and temperature. The nominal elongation was measured during quasi-static tensile testing and during an initial loading step in fatigue testing in which the tensile force was gradually increased to the center of the oscillation of cyclic loadings.

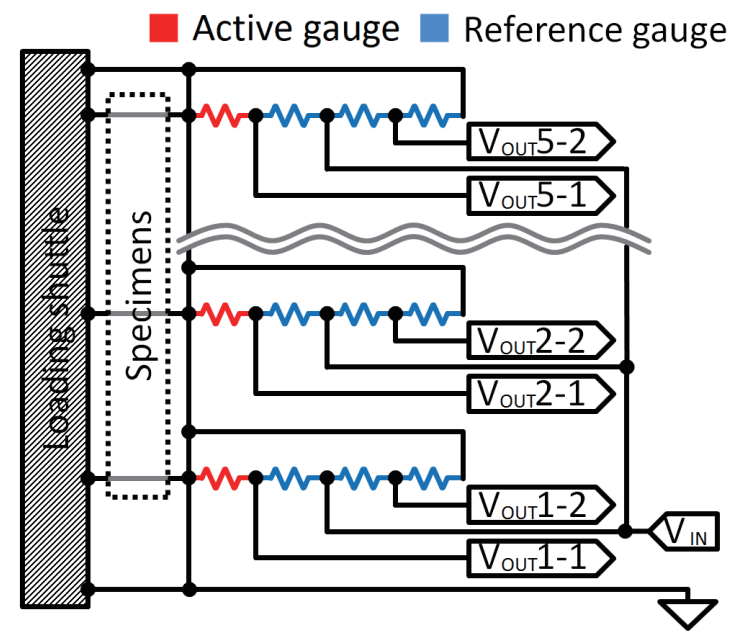

Fig. 5. (Color online) Circuit diagram for parallel testing using single de voltage supply. 


\section{Experiments}

\subsection{Force sensor calibration}

To calibrate the integrated strain gauge, a calibration chip was designed and fabricated on the same wafer with the parallel testing chip. The calibration chip had one specimen device of the same design including the strain gauge. Instead of the loading shuttle of the parallel testing chip, a large paddle was connected at the moving side. The chip was mounted on the piezoelectric stage and the strain gauge was operated in the same way as described in Sect. 3. The paddle was glued to a probe connected to a load cell with a capacity of $500 \mathrm{mN}$ (LTS-50GA, Kyowa Electric Instruments), as shown in Fig. 6. The tensile force was applied with a bridge voltage of $0.5 \mathrm{~V}$, and the bridge output was amplified with a gain of 500 using a de amplifier (DA-510B, Kyowa Electric Instruments) and then acquired using a personal computer.

\subsection{Parallel tensile testing}

The parallel tensile testing of the five-specimen array was conducted using the testing chip, where $0.25 \mathrm{~V}$ was supplied to the bridge. Tensile testing was conducted in laboratory air at a pull rate of $0.07 \mu \mathrm{m} / \mathrm{s}$ of the piezoelectric stage. During the testing, the nominal elongation of the specimen located at the center of the testing chip was monitored.

\section{Results}

\subsection{Force sensor calibration}

Figure 7 shows the strain gauge output, force, and nominal elongation of the calibration device against the position of the piezoelectric stage. The strain gauge exhibited a linear output against the force, as shown in Fig. 7(a). The strain gauge output before amplification was calculated to be $-0.023 \mathrm{mV} / \mathrm{V}$ at $1 \mathrm{mN}$. The noise level of the amplified bridge output was
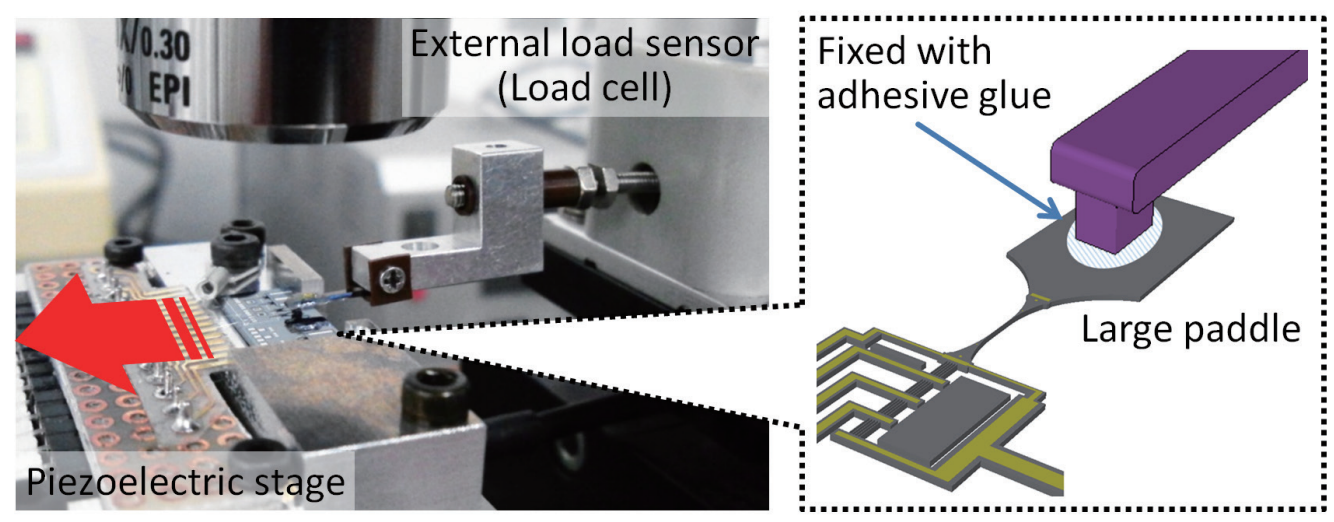

Fig. 6. (Color online) Setup for calibration test. 


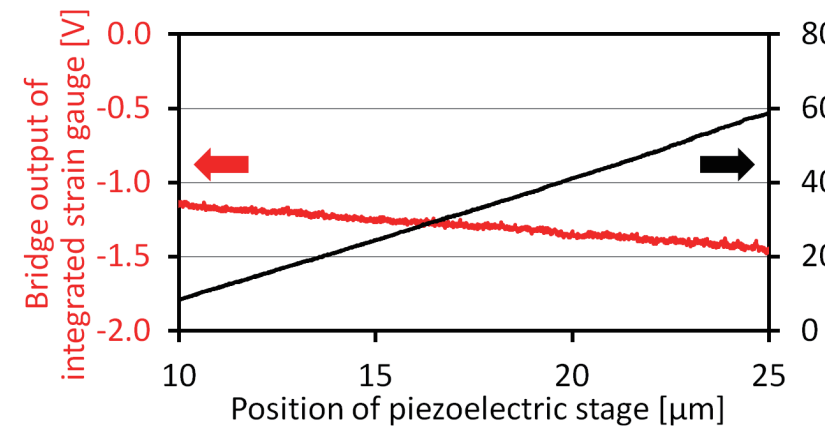

(a)

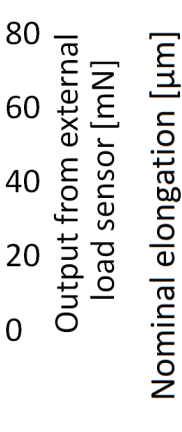

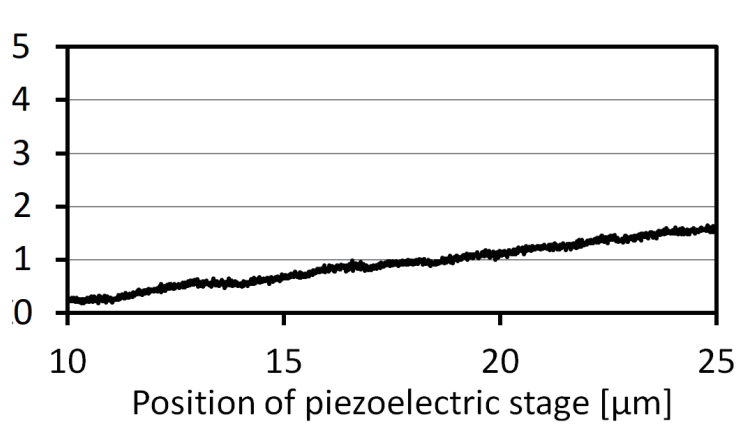

(b)

Fig. 7. (Color online) Calibration results. (a) Amplified bridge output and force measured by the load cell and (b) nominal elongation measured by DIC as a function of the piezoelectric stage position.

$11 \mathrm{mVrms}$, which was equivalent to the $0.02 \%$ strain of the parallel portion of the specimen. Because the fracture strain of silicon microstructures was estimated to be larger than $1 \%$, the small noise level ensured that the method was sufficiently applicable for force monitoring on parallel testing.

As shown in Fig. 7(b), the measured nominal elongation exhibited a linear increase against the stage displacement. The linearity of the strain gauge was confirmed in the range of the nominal elongation to $2 \mu \mathrm{m}$. The nominal elongation was equivalent to a tensile stress of 1.56 GPa at the parallel portion of the specimen, calculated by finite element method (FEM) analysis using CoventorWare.

\subsection{Parallel tensile testing}

Figure 8 shows the amplified bridge outputs from the five specimens and the measured nominal elongation of the center specimen as a function of the position of the piezoelectric stage. The plots indicate that the loading pin contacted the testing chip at $7.5 \mu \mathrm{m}$ of the stage position and specimen fracture occurred at $19.5 \mu \mathrm{m}$, as indicated by the sudden changes in the plots. All specimens in the device seemed to fracture at the same moment, which indicates that the strength difference among the specimens was small enough to cause linked fractures.

Figure 9 shows a fractured specimen on the testing device that exhibits a typical shape of brittle fracture under tensile stress with cleavage planes on (111) planes. The fracture ultimately initiated from damage on the lower part of the sidewall, which was related to the fact that the surface morphology on the lower part was worse owing to etching damage caused during the deep RIE. The tensile strength was calculated as $1.5 \mathrm{GPa}$ by FEM analysis from the measured nominal elongation. The integrated force sensors were not used, since the sensitivity obtained from the calibration test could not be used because of the coupling between sensors described below.

As shown in Fig. 8(a), the amplified bridge outputs show different offset values and the polarity of the output of specimen 1 (at the left end) was opposite to the others. Figure 10 shows the absolute values of the output voltage changes, and the sensitivities of the force sensors 


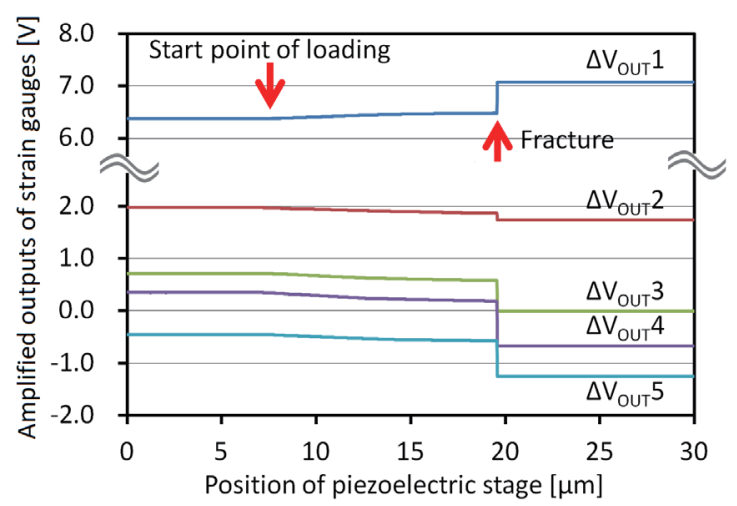

(a)



(b)

Fig. 8. (Color online) Parallel tensile testing results. (a) Measured bridge outputs of five specimens and (b) nominal elongation of specimen at center as a function of piezoelectric stage position.

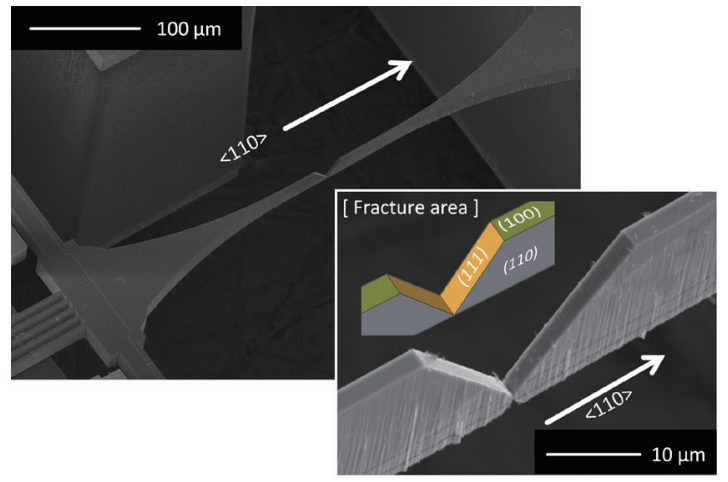

Fig. 9. (Color online) Fracture surface of specimen subjected to parallel tensile testing.

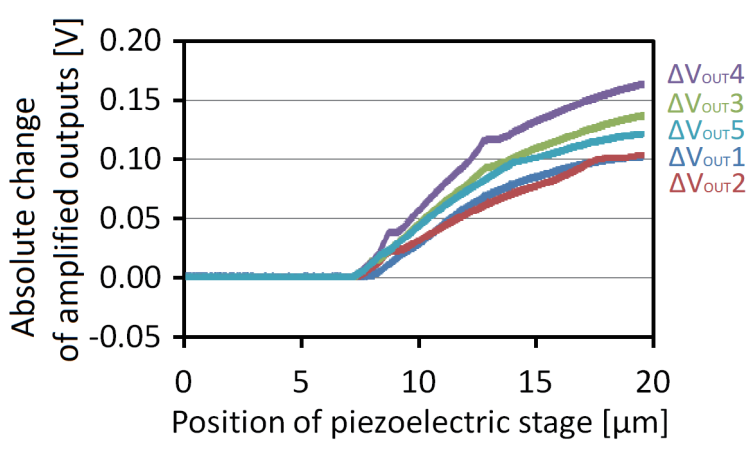

Fig. 10. (Color online) Absolute value plots of changes in bridge outputs on parallel tensile testing.

differed by about $30 \%$. This is not expected, since the five specimens and sensors have identical designs, and the force and stress applied in the device should be the same among them. In order to understand the output, the piezoresistive effect was analyzed using the piezoresistive analysis function of CoventorWare. The whole testing chip was modeled and the pressure equivalent to the fracture strength of the specimen was applied on the top side of the hole of a moving shuttle. Figure 11 shows the electrical potential distribution without loading. The distribution indicates that the resistance of the aluminum wirings is not negligible and the actual bridge voltage on each force sensor is much smaller and different. Both ends of the force sensors that should be biased at $0 \mathrm{~V}$ have non-zero biases and different voltages because of the wiring resistance between them. The simulated offset on the amplified voltage difference agreed well with the measured values shown in Fig. 7(a). The non-zero potential of the moving shuttle also affected the output. Since there was a potential difference between the ends of the tensile specimens, it also affected the amplified output. 


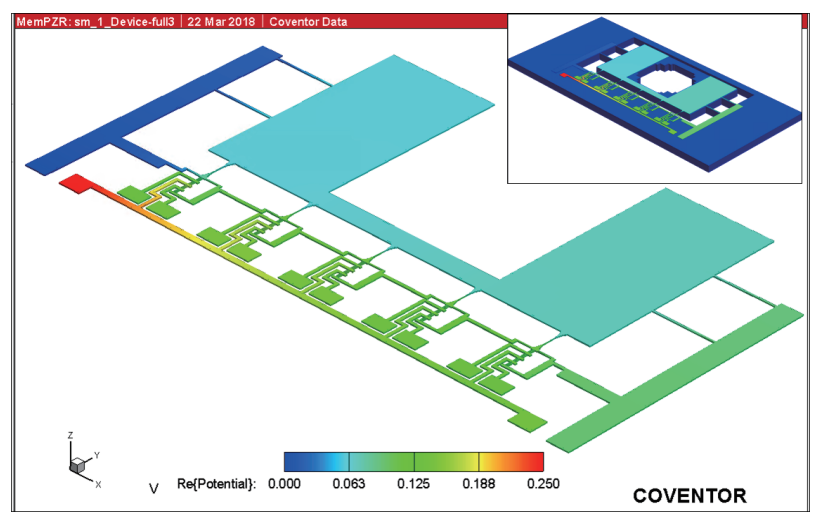

Fig. 11. (Color online) Electrical potential of testing chip on parallel tensile testing. No force is applied in the loading shuttle. Only the device layer is shown. (inset) Whole chip with handle layer.

Figure 12 shows the calculated amplified outputs of the force sensors against the stress on the specimen. The output shows the nonlinearity similar to the measured output, which is caused by the bridge voltage change due to the resistance change itself and the coupling between the other sensors. However, the output is much larger than the measured one and all the outputs show the same polarity. The smaller measured output could be caused by the higher resistance of the aluminum film. When we assume a tenfold higher resistivity of the aluminum film, the bridge voltage becomes smaller. However, the different polarity of the output has not been obtained in the analysis. The nonlinearity in the piezoresistive effect could be the reason, since the tensile stress on the specimen is larger than the reported $1 \mathrm{GPa}^{(21)}$

\section{Discussion}

The developed parallel tensile testing device succeeded in applying tensile force in the five specimens and fracturing the specimens at $1.5 \mathrm{GPa}$. The stage displacement required for fracturing was $12 \mu \mathrm{m}$. With this device and setup, the maximum loading frequency was estimated as $110 \mathrm{~Hz}$, considering the frequency response of the piezoelectric stage shown in Fig. 4. Fatigue testing at $110 \mathrm{~Hz}$ can achieve $10^{8}$ cycles in about 10 days, which means that 20 samples will be tested in 6 weeks.

However, the displacement range was larger than designed. The nominal elongation of the parallel portion of the specimen was estimated to be about $1.1 \mu \mathrm{m}$ under a fracture stress of 1.5 $\mathrm{GPa}$. For the analysis of the relationship between the stage displacement and the deformation of the testing device, the position changes of the two reference points for DIC were plotted, as shown in Fig. 13(a). The measurement points of the displacement in the shuttle, $d_{\text {shuttle, }}$, and that in the gauge, $d_{\text {gauge, }}$ are indicated in Fig. 3(b). The gauge side point should represent the elongation of the gauge, but it also includes the contribution of the displacement of the chip due to the finite stiffness of the alignment stage. The loading shuttle side point represents the motion of the loading pin. Ideally, the motion should be equal to the displacement of the piezoelectric stage, but there is a difference caused mainly by the deformation of the loading 


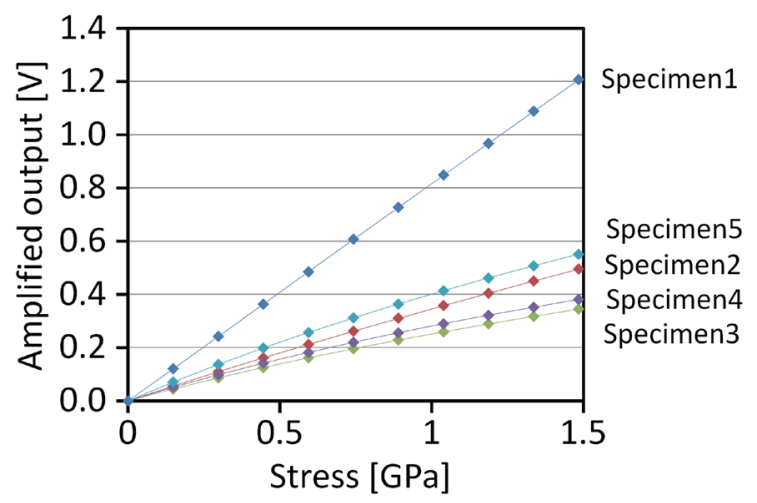

Fig. 12. (Color online) Amplified bridge outputs as a function of maximum Mieses stress calculated by FEM analysis.

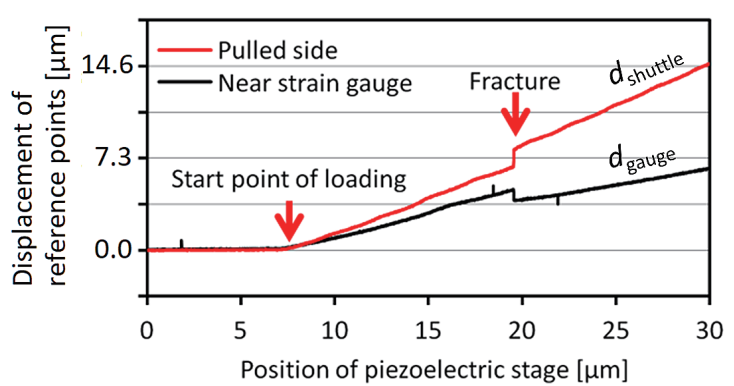

(a)

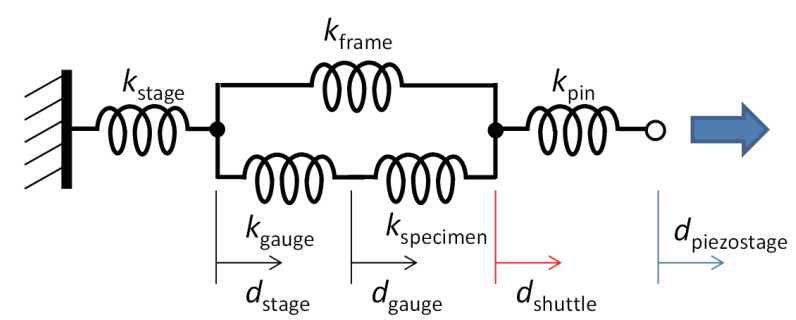

(b)

Fig. 13. (Color online) (a) Displacements of two reference points on specimen at center during parallel tensile testing as a function of the piezoelectric stage position. (b) Equivalent mechanical model showing stiffness of testing setup.

pin. The lumped parameter model of the setup is shown in Fig. 13(b). The total stiffness of the setup before specimen fracture is described as

$$
\frac{1}{k_{t}}=\frac{1}{k_{\text {stage }}}+\frac{1}{k_{\text {frame }}+5 \times \frac{1}{\frac{1}{k_{\text {gauge }}}+\frac{1}{k_{\text {specimen }}}}}+\frac{1}{k_{\text {pin }}},
$$

where the subscripts of the stage, frame, gauge, specimen, and pin indicate the manual alignment stages, supporting beams, strain gauge, specimen, and loading pin, respectively. The slopes of each displacement before and after specimen fracture are fitted to the equation, assuming that the specimen stiffnesses $k_{\text {specimen }}$ are 46.7 and $0 \mathrm{kN} / \mathrm{m}$, respectively. The specimen stiffness was calculated by dividing the fracture force with the measured elongation $\left(d_{\text {shuttle }}-d_{\text {gauge }}\right)$ at fracture. The fracture force was obtained by FEM using the bulk Young's modulus. The other four stiffnesses were optimized so that the calculated displacements at 
Table 3

Calculated stiffnesses of components of test setup.

\begin{tabular}{cccc}
\hline$k_{\text {stage }}$ & $k_{\text {frame }}$ & $k_{\text {gauge }}$ & $k_{\text {pin }}$ \\
\hline 143 & 115 & 368 & 120 \\
\hline Unit: $\mathrm{kN} / \mathrm{m}$ & &
\end{tabular}

the gauge and shuttle fit to the measured displacements using the solver function of Microsoft Excel. The obtained stiffnesses are listed in Table 3. The gauge stiffness was $368 \mathrm{kN} / \mathrm{m}$, which shows good agreement with the theoretical value of $464 \mathrm{kN} / \mathrm{m}$. Because of the unexpected low stiffness of the manual stages and loading pin, the total displacement required for testing is larger. We need to increase the stiffnesses of the stage and pin.

In addition, on fracturing of one of the five specimens, the stress on a remaining specimen increases by $12 \%$. The large increase is the main reason for the linked fracture. This increase can be reduced by increasing the ratio of the stiffness of the supporting beam to that of the specimen. If the ratio is 10 times higher, the increase will decrease to $2 \%$.

\section{Conclusions}

We have proposed a parallel tensile testing device integrated with piezoresistive strain gauges to realize the fatigue testing of a number of specimens in a short time. Parallel tensile testing on the testing chip clearly exhibited loading initiation and fracture occurrence. The required displacement of the piezoelectric stage for fracturing specimens was about $12 \mu \mathrm{m}$ because of its high stiffness realized with the integration of the strain gauges, which would realize tensile-mode fatigue testing with a loading frequency of $110 \mathrm{~Hz}$. By using a loading frequency of $110 \mathrm{~Hz}$, fatigue testing up to $10^{8}$ cycles can be achieved in about 10 days and, in combination with parallel testing, would reduce the total testing time significantly. We plan to fabricate the testing device with revised wiring to improve the testing accuracy and conduct tensile-mode fatigue testing.

\section{Acknowledgments}

This work was supported by a Grant-in-Aid for the Japan Society for the Promotion of Science Fellows. Part of this work was supported by the Kyoto University Nano Technology Hub in the "Nanotechnology Platform Project" sponsored by the Ministry of Education, Culture, Sports, Science and Technology (MEXT), Japan.

\section{References}

1 T. Tsuchiya, M. Hirata, N. Chiba, R. Udo, Y. Yoshitomi, T. Ando, K. Sato, K. Takashima, Y. Higo, Y. Saotome, H. Ogawa, and K. Ozaki: J. Microelectromech. Syst. 14 (2015) 1178.

2 K. Sato, T. Yoshioka, T. Ando, M. Shikida, and T. Kawabata: Sens. Actuators, A 70 (1998) 148.

3 T. Namazu, Y. Isono, and T. Tanaka: J. Microelectromech. Syst. 9 (2000) 450.

4 T. Tsuchiya, O. Tabata, J. Sakata, and Y. Taga: J. Microelectromech. Syst. 7 (1998) 106. 
5 T. Yi, L. Li, and C. J. Kim: Sens. Actuators, A 83 (2000) 172.

6 M. S. Gaither, F. W. Delrio, R. S. Gates, E. R. Fuller Jr., and R. F. Cook: Scr. Mater. 63 (2010) 422.

7 S. Nakao, T. Ando, M. Shikida, and K. Sato: J. Micromech. Microeng. 18 (2008) 015026.

8 T. Tsuchiya, T. Ikeda, A. Tsunematsu, K. Sugano, and O. Tabata: Sens. Mater. 22 (2010) 1.

9 T. Fujii, K. Sudoh, S. Sakakihara, M. Naito, S. Inoue, and T. Namazu: Jpn. J. Appl. Phys. 52 (2013) 110118.

10 C. L. Muhlstein, S. B. Brown, and R. O. Ritchie: Sens. Actuators, A 94 (2001) 177.

11 T. Ikehara and T. Tsuchiya: J. Microelectromech. Syst. 21 (2012) 830.

12 H. Kahn, R. Ballarini, J. J. Bellante, and A. H. Heuer: Science 298 (2002) 1215.

13 T. Namazu and Y. Isono: J. Microelectromech. Syst. 18 (2009) 129.

14 T. Tsuchiya, Y. Yamaji, K. Sugano, and O. Tabata: Exp. Mech. 50 (2010) 509.

15 T. Ando, M. Shikida, and K. Sato: Sens. Actuators, A 93 (2001) 70.

16 J. Bagdahn and W. N. Sharpe Jr.: Sens. Actuators, A 103 (2003) 9.

17 S. Kamiya, S. Amaki, T. Kawai, N. Honda, P. Ruther, J. Gaspar, and O. Paul: J. Micromech. Microeng. 18 (2008) 095023.

18 H. Kapels, R. Aigner, and J. Binder J: IEEE Trans. Electron Dev. 47 (2000) 1522.

19 A. Uesugi, Y. Hirai, T. Tsuchiya, and O. Tabata: Proc. 28th Int. Microprocesses and Nanotechnology Conf. (JJAP, 2015) 13P-11-124L.

20 Y. Kanda: IEEE Trans. Electron Devices 29 (1982) 64.

21 J. Gaspar, J. Gutmann, B. Lemke, and O. Paul: Proc. IEEE Int. Conf. Micro Electro Mechanical Systems (IEEE, 2011) 469.

\section{About the Authors}

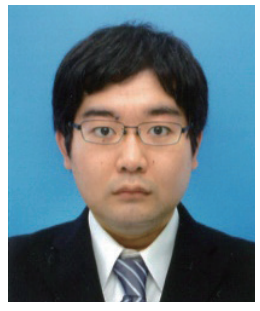

Akio Uesugi received his B.S., M.S., and Ph.D. degrees from Kyoto University, Japan, in 2011, 2013, and 2016, respectively. From 2016 to 2018, he was a postdoctoral researcher at Aichi Institute of Technology, Japan. Since 2018, he has been an assistant professor at Kobe University. His research interests are in the mechanical characterization of microstructures used for MEMS and sensors.

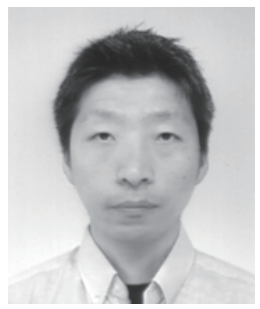

Yoshikazu Hirai received his B.S. and M.S. degrees from Ritsumeikan University, Japan, in 2002 and 2004, respectively, and his Ph.D. degree from Kyoto University, Japan, in 2007, all in mechanical engineering. He was a postdoctoral researcher at the Graduate School of Engineering, Kyoto University. In 2009, he joined the Advanced Biomedical Engineering Research Unit, Kyoto University. Since 2013, he has been an assistant professor at the Department of Micro Engineering, Kyoto University. He is also currently an adjunct assistant professor at the Institute for Integrated Cell-Material Sciences, Kyoto University. His current research interests include 3-D micro/nanofabrication, chip-scale atomic devices, and microfluidic devices for biomedical engineering. 


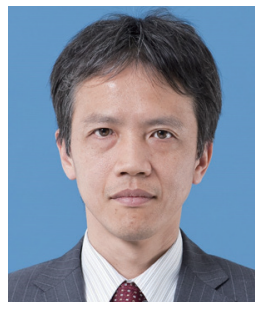

Toshiyuki Tsuchiya received his B.S. and M.S. degrees from the University of Tokyo, Japan, and his Ph.D. degree from Nagoya University, Japan, in 1991, 1993, and 2002, respectively. He worked at Toyota Central Research and Development Laboratories from 1993 to 2004. In 2004, he joined Kyoto University as an associate professor and now belongs to the Department of Micro Engineering, Kyoto University, Japan. He is currently engaged in the research of silicon surface micromachining, its application in MEMS, the mechanical property evaluation of micromaterials, and the reliability of MEMS devices.

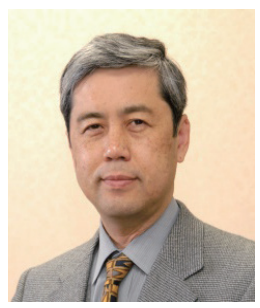

Osamu Tabata received his M.S. and Ph.D. degrees from Nagoya Institute of Technology, Japan, in 1981 and 1993, respectively. In 1981, he joined the Toyota Central Research and Development Laboratories, Inc., Japan. In 1996, he joined the Department of Mechanical Engineering, Ritsumeikan University, Japan. In 2003, he joined the Department of Mechanical Engineering, Kyoto University, Japan. Since April 2005, he has been a professor in the Department of Micro Engineering, Kyoto University. He is currently engaged in research on micro/nanoprocesses, MEMS, DNA nanotechnology, and micro/nanosystem synthetic engineering. He was a guest professor of the Institute of Microsystem Technology, University of Freiburg, Germany from September to December 2000, a guest professor of ETH Zurich, Switzerland from January to March 2001, a visiting senior international scientist of the Chinese Academy of Science in 2010, a senior research fellow at Freiburg Institute for Advanced Studies (FRIAS) from May 2010 to 2013 October, and a guest Professor of Huazong University of Science and Technology, China from July 2011 to July 2014. 